Artículo

\title{
Dinámica de adopción de buenas prácticas de producción de miel en la península de Yucatán, México
}

\section{Dynamics of adoption of good practices in honey production in the Peninsula of Yucatan, Mexico}

Enrique Genaro Martínez-González ${ }^{\mathrm{a}}$,

Hortencia Arroyo-Pozos ${ }^{\mathrm{a}}$,

Norman Aguilar-Gallegos ${ }^{\mathrm{a}}$,

José María García Álvarez-Coque ${ }^{\mathrm{b}}$,

Vinicio Horacio Santoyo-Cortés ${ }^{\mathrm{a}}$,

Jorge Aguilar-Ávila ${ }^{{ }^{*}}$

${ }^{a}$ Centro de Investigaciones Económicas, Sociales y Tecnológicas de la Agroindustria y la Agricultura Mundial (CIESTAAM) de la Universidad Autónoma Chapingo (UACh). México.

b Departamento de Economía y Ciencias Sociales, Universidad Politécnica de Valencia. Valencia, España.

Autor de correspondencia jaguilar@ciestaam.edu.mx

\footnotetext{
- Resumen:

Los objetivos fueron analizar los cambios en la adopción de buenas prácticas de producción de miel (BPPM) en productores apícolas de la Península de Yucatán, México, antes y después de haber recibido asesoría técnica y capacitación; así como la identificación de factores que inciden en la adopción de dichas prácticas. Considerando 31 BPPM agrupadas en siete categorías, se calculó el índice de adopción de BPPM (InABPPM). Se analizó información de 8,074 apicultores en dos momentos (línea base y línea final), para los estados de Yucatán $(n=3,840)$, Campeche $(n=3,525)$ y Quintana Roo $(n=709)$. Los apicultores se clasificaron de acuerdo al número de colmenas que poseían en: pequeños (1-22, $24 \%$ ), medianos (23-35,
} 
$27 \%)$ y grandes (36-400, $49 \%)$. El InABPPM global presentó un incremento significativo $(P<0.01)$ de $16.8 \%$ en un periodo de ocho meses. Además, se encontraron diferencias significativas $(P<0.01)$ en las categorías de ubicación e instalación del apiario, sanidad apícola, cosecha, personal en campo y programa de limpieza e higiene. No se encontraron diferencias significativas para las categorías de alimentación artificial y materiales para la protección y ahumado de la colmena $(P>0.05)$. Este estudio destaca la importancia de programas para la gestión y difusión de BPPM, con la finalidad de impulsar la actividad apícola y acceder al mercado internacional cumpliendo con las normas de calidad requeridas. Los resultados sugieren impulsar la adopción de las BPPM de proceso, y posteriormente orientar la inversión de los apoyos púbicos en aquellos apiarios con mejor desempeño en cuanto al incremento en sus niveles de adopción.

- Palabras clave: Extensionismo agrícola, Apicultura, Certificación, Innovación, Inocuidad.

\begin{abstract}
- Abstract:
The objectives were to analyse the changes in the adoption of good honey production practices (BPPM, in Spanish) in apicultural producers of the Yucatan Peninsula, Mexico, before and after the producers had received technical advice and training; as well as identifying factors that influence the adoption of such practices. A total of 31 BPPM were considered and grouped into seven categories, the BPPM adoption index (InABPPM, in Spanish) was figured up. Information from 8,074 beekeepers in two stages (baseline and final line), for the states of Yucatan ( $\mathrm{n}=3,840)$, Campeche $(\mathrm{n}=3,525)$, and Quintana Roo $(\mathrm{n}=709)$, was analysed. Beekeepers were classified by the number of hives they owned, in three groups: small $(1-22,24 \%)$, medium $(23-35,27 \%)$, and large (36-400, $49 \%)$. The average InABPPM had a significant increase $(P<0.01)$ of $16.8 \%$ in a period 8 mo. Additionally, significant differences $(P<0.01)$ were found in the categories of location and installation of apiary, apicultural health, harvesting, field staff, and cleaning and hygiene program. No significant differences were found for the categories of artificial feeding and materials for the hive protection and smoking $(P>0.05)$. This study highlights the relevance of programs for managing and disseminating of BPPM to promote the beekeeping activity and access to the international market complying with the required quality standards. The results suggest promoting the adoption of process BPPM and later guide the investment of public support in those apiaries with better performance in terms of increasing in adoption levels.
\end{abstract}

Key words: Agricultural extension, Beekeeping, Certification, Innovation, Safety.

Recibido el 15/02/2017.

Aceptado el 5/07/2017. 


\section{N Introducción}

La apicultura mexicana es una actividad tradicional reconocida a nivel internacional. México está entre los principales productores y exportadores de miel de alta calidad; en 2015 exportó alrededor del $65 \%$ de su producción ${ }^{(1)}$. Además, el nivel de competitividad de la apicultura mexicana en el mercado internacional es alto, sólo superado por China y Argentina en indicadores de trazabilidad y especialización internacional ${ }^{(2)}$.

A nivel global, se reconoce que la apicultura proporciona grandes beneficios al medio ambiente; dado que es una actividad compatible prácticamente con todo tipo de ecosistema, favoreciendo la polinización de la flora, pero su desarrollo y productividad requiere de agroecosistemas sustentables ${ }^{(3)}$. Sin embargo, la apicultura también enfrenta algunos retos, como son: el uso de pesticidas en las zonas aledañas a los apiarios, la contaminación por transgénicos, el crecimiento de las zonas urbanas, entre otros, los cuales frenan su crecimiento y desarrollo ${ }^{(3,4)}$.

Por otra parte, la Secretaria de Agricultura, Ganadería, Desarrollo Rural, Pesca y Alimentación (SAGARPA) define a las buenas prácticas pecuarias en producción como un "conjunto de recomendaciones generales enfocadas a garantizar la calidad e inocuidad de los alimentos, reduciendo los riesgos físicos, químicos y microbiológicos durante el proceso de obtención y cosecha"(5).

Uno de los aspectos de la producción de miel en México es que se sabe que se realiza principalmente de forma convencional, situación presente en la región sureste del país, la cual aporta una tercera parte de la producción nacional y que, además, tiene características florísticas únicas para la obtención de un producto de buena calidad ${ }^{(6)}$. En este contexto, la SAGARPA a través del Programa Estratégico para el Desarrollo Rural Sustentable de la Región Sur-Sureste de México (Proyecto Transversal Trópico Húmedo), implementó del 2011 al 2013 una iniciativa para el desarrollo de capacidades, innovación tecnológica y extensionismo rural, encaminadas a la difusión y adopción de buenas prácticas de producción de miel (BPPM), con la finalidad de mejorar la competitividad de los apicultores, a través de un modelo de gestión de la innovación llamado Agencias de Gestión de la Innovación para el Desarrollo de Proveedores (AGI-DP) ${ }^{(7)}$.

Bajo este contexto, las BPPM se consideraron como innovaciones en el sector apícola, mismas que adoptan los apicultores para mejorar sus sistemas de producción. La adopción mide el resultado de la decisión de los productores de usar o no una innovación (tecnología 
principalmente) determinada en el proceso de producción ${ }^{(8)}$. Por su parte, Rogers ${ }^{(9)}$, atribuye la adopción de innovaciones a sus características intrínsecas, y por tanto, se puede dar la reinvención, que es el grado en que una innovación es modificada por el usuario en el proceso de adopción e implementación.

Los factores que influyen en la adopción de prácticas e innovaciones en el sector agropecuario son múltiples, algunos asociados a la presencia de diversos actores ${ }^{(10,11)}$, otros a diferentes estilos de producción y combinaciones de tecnologías y prácticas ${ }^{(12)}$, a los cambios a nivel institucional ${ }^{(13,14)}$, a la complejidad y adaptabilidad de las innovaciones mismas $^{(9)}$, entre otros ${ }^{(15,16)}$. Sin embargo, uno de los factores que siempre está presente es la participación de agentes externos, principalmente los relacionados a los servicios de extensión agrícola.

Bajo este panorama, en este estudio se analizan los cambios en la adopción de BPPM por parte de productores apícolas de la Península de Yucatán, México; antes y después de haber recibido asesoría técnica y capacitación por parte de las AGI-DP, a través del cálculo del Índice de adopción de buenas prácticas de producción de miel (InABPPM), así como la identificación de factores que inciden en la adopción de dichas prácticas, con la finalidad de generar información para el diseño de estrategias de apoyo a la apicultura.

\section{N Material y métodos V}

La investigación se desarrolló en la Península de Yucatán, México, que comprende los estados de Yucatán, Campeche y Quintana Roo. Se utilizó información obtenida en dos momentos: encuesta de línea base (ELB) y encuesta de línea final (ELF), entre mayo y diciembre de 2011. Se recabó información de 3,840 apicultores en Yucatán, 3,525 en Campeche y 709 en Quintana Roo.

Con base en lo establecido por la SAGARPA ${ }^{(5)}$ a través del Programa Nacional para el Control de Abeja Africana, se construyó un catálogo de 31 BPPM agrupadas en siete categorías: ubicación e instalación del apiario (categoría A), alimentación artificial (B), sanidad apícola (C), materiales para la protección y ahumado de la colmena (D), cosecha (E), personal en campo $(\mathrm{F})$, y programa de limpieza e higiene $(\mathrm{G})$, tal como se muestra en el Cuadro 1. De las encuestas realizadas se obtuvo una base de datos con 16,148 observaciones: 8,074 de ELB y 8,074 de ELF. Los apicultores se clasificaron por el número de colmenas en: pequeños $(1-22$ colmenas, $24 \%)$ medianos $(23-35,27 \%)$ y grandes $(36-400,49 \%)$, 
considerando lo propuesto por otros autores en estudios previos en la región ${ }^{(17)}$ y en otros estados del país ${ }^{(18)}$.

Cuadro 1: Catálogo de buenas prácticas $(\mathrm{BP})$ de producción de miel por categoría

Categoría Buena práctica de producción de miel

BP01 Entorno del apiario; BP02 Apiario limpio de malezas; BP03 Uso de bebederos; BP04 Material adecuado del bebedero; A. Ubicación e BP05 Bebederos limpios; BP06 Colmenas sobre base; BP07 instalación del apiario Distancia a zonas habitadas; BP08 Conocimiento sobre la aplicación productos químicos.

B. Alimentación artificial

C. Sanidad apícola

D. Materiales para protección y ahumado de colmena

E. Cosecha

F. Personal en campo

G. Programa de limpieza e higiene
BP09 Proporciona alimentación artificial; BP10 Insumo permitido por la SAGARPA como fuente de energía; BP11 Insumo permitido por la SAGARPA como complemento; BP12 Momento en que suspende la alimentación.

BP13 Frecuencia de revisión de las colmenas; BP14 Registro en bitácoras; BP15 Productos autorizados por la SAGARPA para prevenir las enfermedades; BP16 Productos autorizados por la SAGARPA para controlar enfermedades; BP17 Control de la varroasis emplea productos autorizados por SAGARPA; BP18 Época de aplicación de productos químicos; BP19 Flameo de la cuña.

BP20 Material para cubrir la colmena; BP21 Condiciones del equipo; BP22 Material o actividad para desalojar las colmenas.

BP23 Porcentaje de operculación de alzas; BP24 Material utilizado para desalojar.

BP25 El personal conoce BPPM; BP26 Tiene bitácora de higiene del personal de campo; BP27 Vestimenta limpia; BP28 Vestimenta es de uso exclusivo.

BP29 Programa de procedimientos sobre higiene y limpieza personal; BP30 Procedimientos higiene y limpieza de equipo de protección; BP31 Procedimientos para la limpieza de los utensilios y recipientes.

Fuente: SAGARPA et $a l^{(5)}$. 


\section{- Indicadores utilizados}

El indicador utilizado para cada momento fue el InABPPM, que es una adaptación del Índice de adopción de innovaciones propuesto por Muñoz et al ${ }^{(19)}$. De esta forma el InABPPM refiere la capacidad innovadora del apicultor, a mayor InABPPM mayor es el nivel de innovación del apicultor y viceversa. Este índice toma valores de entre 0 y 1 , se calcula considerando el número de BPPM que realiza el productor en un momento determinado, respecto al número de prácticas por categoría definidas en el catálogo respectivo.

$$
\text { InABPPM }=\frac{\text { Número de BPPM realizadas por el productor }}{\text { Número de prácticas por categoría }}
$$

Al final, el InABPPM global se obtiene promediando el nivel de adopción de todas las categorías.

\section{- Análisis de la información •}

La información se analizó en tres niveles. En primera instancia el cambio en la adopción de BPPM entre las diferentes categorías del índice y momentos de evaluación (ELB y ELF); para ello se realizó una prueba de t para muestras relacionadas para el InABPPM en los dos momentos, así como análisis de varianza mediante un modelo completamente aleatorio para detectar diferencias por tipo de apicultor según el tamaño del apiario y estados participantes.

En un segundo nivel de análisis se consideró el incremento en el InABPPM por cada categoría, para lo cual también se realizó un análisis de varianza para encontrar diferencias entre los tipos de apicultores, según el número de colmenas.

Finalmente, se identificaron algunas variables internas y externas a la producción apícola que pudieran influir en el cambio en la adopción de innovaciones. Para ello se incluyeron nuevas variables como la zona de ubicación del apiario, según el grado de ruralidad; esto con base en la densidad de la población ${ }^{(20)}$, considerando como "rural" si la densidad de población es menor de 150 habitantes por $\mathrm{km}^{2}$, a mayor población se consideró "urbana"; la información de la población se obtuvo del censo de población y vivienda $2010^{(21)}$. 
Considerando el conjunto de variables (internas y externas al apiario), se especificó un modelo de regresión lineal múltiple para explicar el cambio en el InABPPM:

$$
\begin{gathered}
Y=\beta_{0}+\beta_{1} X_{1}+\beta_{2} X_{2}+\beta_{3} X_{3}+\beta_{4} X_{4}+\beta_{5} X_{5}+\beta_{6} X_{6}+\beta_{7} X_{7}+\beta_{8} X_{8}+\beta_{9} X_{9}+\beta_{10} X_{10}+ \\
\beta_{11} X_{11}+\beta_{12} X_{12}+\varepsilon_{i}
\end{gathered}
$$

Donde:

Y= cambio en el índice de adopción de BPPM;

$\beta_{0}=$ coeficiente constante de regresión del modelo;

$\mathrm{X}_{1}=$ zona o territorio (rural $=0$, urbano $=1$ );

$\mathrm{X}_{2}=$ tamaño A del sistema de producción apícola;

$\mathrm{X}_{3}=$ tamaño $\mathrm{B}$ del sistema de producción apícola;

$\mathrm{X}_{4}=$ sistema de identificación del apiario $($ no tiene $=0$, tiene $=1)$;

$\mathrm{X}_{5}=$ género de quien atiende el apiario $($ masculino $=0$, femenino $=1)$.

Cambios en los niveles de adopción de innovaciones por categoría;

$\mathrm{X}_{6}=$ ubicación e instalación del apiario;

$\mathrm{X}_{7}=$ alimentación artificial;

$\mathrm{X}_{8}=$ sanidad apícola;

$\mathrm{X}_{9}=$ materiales para la protección y ahumado de la colmena;

$\mathrm{X}_{10}=$ cosecha;

$\mathrm{X}_{11}=$ personal en campo;

$\mathrm{X}_{12}=$ programa de limpieza e higiene;

$\varepsilon=$ término error del modelo.

Las variables $\mathrm{X}_{2} \mathrm{y} \mathrm{X}_{3}$ relacionadas con el tamaño de apiario, se construyeron con base en lo propuesto por Montgomery et $a l^{(22)}$, utilizando dos variables indicadoras que en sus combinaciones representan las tres posibles respuestas: $\mathrm{X}_{2}=0 ; \mathrm{X}_{3}=0$, entonces pequeño; $\mathrm{X}_{2}=$ $1 ; X_{3}=0$, entonces mediano; $X_{2}=0 ; X_{3}=1$, entonces grande. Todas las pruebas se realizaron con el software estadístico SAS $9.0^{(23)}$. 


\section{- Adopción de buenas prácticas de producción de miel •}

Comparando el InABPPM global de la ELF con la ELB se presentó un incremento significativo $(P<0.01)$ de $16.8 \%$, lo cual indica el nivel de apropiación que tuvieron los apicultores de las BPPM a través de la intervención del programa de capacitación y asistencia técnica (Figura 1).

Figura 1: Cambios en la adopción de buenas prácticas de producción de miel por categoría y momento de medición en apiculotores de la Península de Yucatán, México

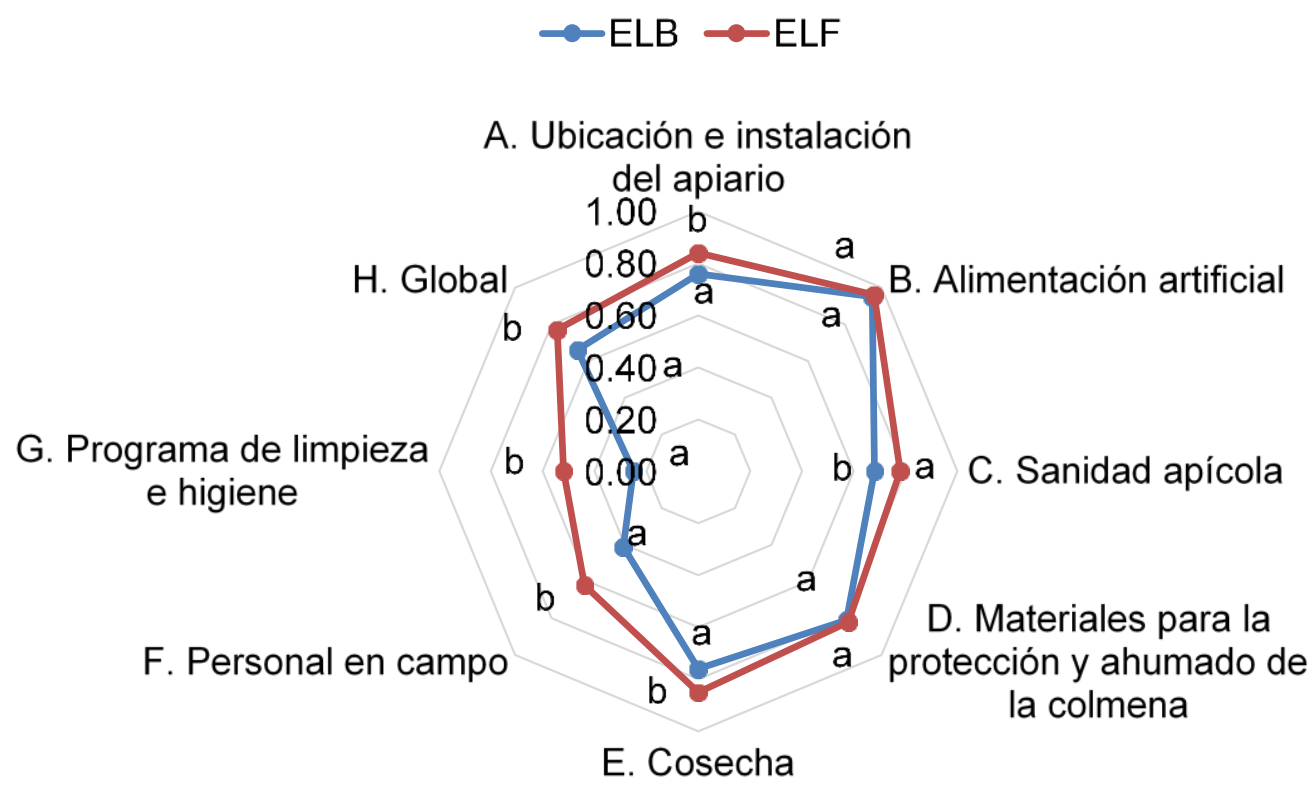

${ }^{\text {ab }}$ Diferentes literales en categorías indican diferencias significativas $(P<0.01)$, de acuerdo con la prueba de $\mathrm{t}$ para muestras relacionadas.

Se encontraron diferencias significativas $(P<0.01)$ en las categorías de ubicación e instalación del apiario (con una tasa de cambio porcentual de $10.8 \%$ ), sanidad apícola (14.9\%), cosecha $(12.1 \%)$, personal en campo (52.4\%) y programa de limpieza e higiene (109.3\%). No se encontraron diferencias significativas $(P>0.10)$ para las categorías de alimentación artificial $(0.4 \%)$ y materiales para la protección y ahumado de la colmena $(1.2 \%)$. 


\section{- Adopción de buenas prácticas de producción de miel por tipo de apicultor •}

Utilizando la tipificación de los apicultores (Cuadro 2) según el tamaño de sus apiarios, las categorías de adopción y, los momentos evaluados (ELB y ELF), los resultados muestran que los apicultores pequeños adoptaron en mayor medida las BPPM en las categorías de ubicación e instalación del apiario, alimentación artificial, sanidad apícola, cosecha, y personal de campo. Así mismo, el análisis de los resultados por categoría de las BPPM muestra que en aquéllas con mayor adopción en la ELB (como alimentación artificial y, materiales para la protección y ahumado de la colmena), el incremento en la adopción en ELF es menor y no presenta diferencias significativas $(P>0.10)$. Es decir, categorías de innovaciones con tasas de adopción altas inicialmente tienden a presentar aumentos marginales en su adopción.

Cuadro 2: Índice de adopción de buenas prácticas de producción de miel (InABPPM) por tipo de apicultor y momento de medición

\begin{tabular}{|c|c|c|c|c|}
\hline \multirow[b]{2}{*}{ Categoría } & & \multicolumn{3}{|c|}{ Tipo de apicultor } \\
\hline & & \multirow{2}{*}{$\frac{\text { Pequeño }}{0.758 \text { a } \mathrm{A}}$} & \multirow{2}{*}{$\begin{array}{l}\text { Mediano } \\
0.759 \text { a A }\end{array}$} & \multirow{2}{*}{$\begin{array}{l}\text { Grande } \\
0.756 \text { a } \mathrm{A}\end{array}$} \\
\hline A: $\quad$ Ubicación e & ELB & & & \\
\hline instalación del apiario & ELF & $0.848 \mathrm{~b} \mathrm{C}$ & $0.833 \mathrm{~b} \mathrm{~A}$ & $0.831 \mathrm{~b} \mathrm{~B}$ \\
\hline \multirow{2}{*}{$\begin{array}{l}\text { B: Alimentación } \\
\text { artificial }\end{array}$} & ELB & 0.951 a A & 0.957 a A & 0.957 a A \\
\hline & ELF & $0.959 \mathrm{~b} \mathrm{~A}$ & 0.957 a A & 0.960 a $\mathrm{A}$ \\
\hline \multirow[t]{2}{*}{ C: Sanidad apícola } & ELB & 0.666 a A & 0.684 a B & 0.691 a C \\
\hline & ELF & $0.779 \mathrm{~b} \mathrm{~A}$ & $0.778 \mathrm{~b} \mathrm{~A}$ & $0.777 \mathrm{~b} \mathrm{~A}$ \\
\hline \multirow{2}{*}{$\begin{array}{l}\text { D: Materiales para la } \\
\text { protección y ahumado } \\
\text { de la colmena }\end{array}$} & ELB & 0.826 a B & 0.803 a A & 0.796 a $\mathrm{A}$ \\
\hline & ELF & 0.835 a C & 0.807 a $\mathrm{A}$ & $0.812 \mathrm{~b} \mathrm{~B}$ \\
\hline \multirow[t]{2}{*}{ E: Cosecha } & ELB & 0.745 a $\mathrm{A}$ & 0.767 a B & 0.788 a C \\
\hline & ELF & $0.856 \mathrm{~b} \mathrm{~A}$ & $0.853 \mathrm{~b} \mathrm{~A}$ & $0.850 \mathrm{~b} \mathrm{~A}$ \\
\hline \multirow[t]{2}{*}{ F: Personal en campo } & ELB & 0.378 a $\mathrm{A}$ & 0.429 a B & 0.456 a C \\
\hline & ELF & $0.618 \mathrm{~b} \mathrm{~A}$ & $0.622 \mathrm{~b} \mathrm{~A}$ & $0.643 \mathrm{~b} \mathrm{~B}$ \\
\hline \multirow{2}{*}{$\begin{array}{ll}\text { G: Programa } & \text { de } \\
\text { limpieza e higiene } & \end{array}$} & ELB & 0.225 a $\mathrm{A}$ & 0.241 a B & 0.287 a C \\
\hline & ELF & $0.486 \mathrm{~b} \mathrm{~A}$ & $0.528 \mathrm{~b} \mathrm{~B}$ & $0.565 \mathrm{~b} \mathrm{C}$ \\
\hline \multirow[t]{2}{*}{ InABPPM global } & ELB & 0.650 a A & 0.664 a B & 0.676 a $\mathrm{C}$ \\
\hline & ELF & $0.769 \mathrm{~b} \mathrm{~A}$ & $0.768 \mathrm{~b} \mathrm{~A}$ & $0.777 \mathrm{~b} \mathrm{~B}$ \\
\hline
\end{tabular}

$\mathrm{ELB}=$ encuesta línea base; $\mathrm{ELF}=$ encuesta línea final. 
ab Diferentes literales indican diferencias $(P<0.01)$ entre momentos de medición (ELB y ELF), por cada tipo de productor.

ABC Diferentes literales indican diferencias $(P<0.01)$ entre tipo de productores, por cada momento de medición.

Con relación al tamaño del apiario, prácticamente en los tres tipos de apicultores hubo cambios significativos $(P<0.01)$ entre la ELB y la ELF, con excepción de categorías como alimentación artificial y, materiales para la protección y ahumado de la colmena. Las categorías en cuestión presentan niveles de adopción altos inicialmente y en ELF sus incrementos son menores.

\section{- Adopción de buenas prácticas de producción de miel por Estado}

Considerando el Estado, momento de evaluación y categorías del InABPPM, existieron diferencias significativas $(P<0.05)$ en el índice de adopción de las tres entidades (Cuadro 3 ). En el estado de Yucatán fue donde el nivel de adopción final fue mayor $(P<0.05)$, seguido de Campeche y después Quintana Roo, explicándose por los incrementos en las categorías de sanidad apícola, cosecha, personal en campo y programa de limpieza e higiene.

Cuadro 3: Índice de adopción de buenas prácticas de producción de miel (InABPPM) por estado y momento de medición

\begin{tabular}{lllll}
\hline \multirow{2}{*}{ Categoría } & & \multicolumn{3}{c}{ Estado } \\
\cline { 3 - 5 } A: Ubicación e instalación del & ELB & 0.805 a C & 0.721 a B & 0.681 a A \\
apiario & ELF & 0.889 b C & 0.790 b A & 0.817 b B \\
\hline B: Alimentación artificial & ELB & 0.955 a B & 0.956 a B & 0.942 a A \\
& ELF & 0.951 a A & 0.965 a B & 0.964 b B \\
\hline C: Sanidad apícola & ELB & 0.672 a B & 0.685 a C & 0.659 a A \\
& ELF & 0.806 b C & 0.764 b B & 0.691 b A \\
\hline D: Materiales para la protección y & ELB & 0.809 a B & 0.795 a A & 0.920 a C \\
ahumado de la colmena & ELF & 0.816 a A & 0.807 b A & 0.928 a B \\
\hline E: Cosecha & ELB & 0.741 a A & 0.780 a B & 0.778 a B \\
& ELF & 0.887 b C & 0.832 b B & 0.783 b A \\
\hline F: Personal en campo & ELB & 0.331 a A & 0.487 a C & 0.456 a B \\
& ELF & 0.664 b C & 0.609 b B & 0.490 b A \\
\hline G: Programa de limpieza e higiene & ELB & 0.203 a B & 0.321 a C & 0.113 a A \\
& ELF & 0.557 b B & 0.547 b B & 0.147 a A \\
\hline InABPPM Global & ELB & 0.645 a A & 0.678 a B & 0.650 a A \\
& ELF & 0.796 b C & 0.759 b B & 0.689 b A \\
\hline
\end{tabular}


ELB= encuesta línea base; $\mathrm{ELF}=$ encuesta línea final.

ab Diferentes literales indican diferencias $(P<0.05)$ entre momentos de medición (ELB y ELF), por cada Estado. ABC Diferentes literales indican diferencias $(P<0.05)$ entre entidades, por cada momento de medición.

Destaca el hecho que en la categoría de alimentación artificial en los tres Estados se observan altos niveles de adopción, aún antes de la implementación del programa de asistencia técnica y capacitación, por lo cual no se dieron incrementos significativos $(P>0.10)$ entre los dos momentos analizados, con excepción del estado de Quintana Roo $(P<0.05)$. Algo similar se presentó en la categoría de materiales para la protección y ahumado de la colmena, donde el cambio fue significativo $(P<0.05)$ solamente en el estado de Campeche.

\section{Incremento en el InABPPM por tipo de apicultor}

Analizando el cambio existente en el InABPPM entre la ELB y ELF por tipo de apicultor (Cuadro 4), se encontró que de manera global dicho cambio fue positivo en los tres tipos de apicultores, con un mayor incremento en los pequeños $(P<0.01)$ con relación a los medianos y grandes. En particular, en las categorías de ubicación e instalación del apiario, sanidad apícola y personal en campo, existieron diferencias significativas entre los pequeños apicultores respecto a los medianos y grandes $(P<0.01)$.

Cuadro 4: Cambio en el índice de adopción de buenas prácticas de producción de miel (InABPPM) por tipo de productor en cada categoría*

\begin{tabular}{|c|c|c|c|}
\hline \multirow[b]{2}{*}{ Categorías } & \multicolumn{3}{|c|}{ Tipo de productor } \\
\hline & Pequeño & Mediano & Grande \\
\hline $\begin{array}{l}\text { A: Ubicación e instalación del } \\
\text { apiario }\end{array}$ & $0.090 \pm 0.003 \mathrm{~b}$ & $0.074 \pm 0.004 \mathrm{a}$ & $0.075 \pm 0.004 \mathrm{a}$ \\
\hline B: Alimentación artificial & $0.008 \pm 0.002 \mathrm{a}$ & $0.000 \pm 0.003 \mathrm{a}$ & $0.002 \pm 0.003 \mathrm{a}$ \\
\hline C: Sanidad apícola & $0.113 \pm 0.003 \mathrm{~b}$ & $0.094 \pm 0.004 \mathrm{a}$ & $0.086 \pm 0.004 \mathrm{a}$ \\
\hline $\begin{array}{l}\text { D: Materiales protección y } \\
\text { ahumado de la colmena }\end{array}$ & $0.009 \pm 0.004 \mathrm{a}$ & $0.004 \pm 0.005 \mathrm{a}$ & $0.016 \pm 0.005 \mathrm{a}$ \\
\hline E: Cosecha & $0.111 \pm 0.005 \mathrm{c}$ & $0.086 \pm 0.006 \mathrm{~b}$ & $0.062 \pm 0.007 \mathrm{a}$ \\
\hline F: Personal en campo & $0.240 \pm 0.005 \mathrm{~b}$ & $0.194 \pm 0.007 \mathrm{a}$ & $0.187 \pm 0.007 \mathrm{a}$ \\
\hline $\begin{array}{l}\text { G: Programa de higiene y } \\
\text { limpieza }\end{array}$ & $0.262 \pm 0.007 \mathrm{a}$ & $0.277 \pm 0.009 \mathrm{a}$ & $0.278 \pm 0.278 \mathrm{a}$ \\
\hline
\end{tabular}




\begin{tabular}{llll}
\hline InABPPM global & $0.119 \pm 0.001 \mathrm{~b}$ & $0.104 \pm 0.003 \mathrm{a}$ & $0.101 \pm 0.003 \mathrm{a}$ \\
\hline
\end{tabular}

* Los cambios en el InABPPM se presentaron en un periodo de ocho meses; los valores del cuadro representan la media \pm desviación estándar.

abc Diferentes literales indican diferencias $(P<0.01)$ entre tipos de apicultores.

En las categorías de alimentación artificial, materiales para la protección y ahumado de la colmena, y programa de higiene y limpieza no se encontraron diferencias $(P>0.1)$. Sólo en la categoría de cosecha, los resultados fueron diferentes para los tres tipos de apicultores $(P<0.01)$.

\section{- Variables internas y externas que influyen en la adopción de innovaciones •}

El modelo de regresión múltiple especificado presenta una bondad de ajuste significativa $(P<$ $0.01)$, lo que sugiere que las variables consideradas en éste, explican los incrementos en el InABPPM (Cuadro 5). Los coeficientes de determinación normal $\left(\mathrm{R}^{2}=0.393\right)$ y ajustado $\left(\dot{\mathrm{R}}^{2}=0.392\right)$ fueron muy similares, lo que indica que el modelo no está sobre ajustado; sin embargo, presenta un bajo poder predictivo.

Cuadro 5: Variables que influyen en la adopción de buenas prácticas de producción de miel, por apicultores de la Península de Yucatán, México

\begin{tabular}{lcccc}
\hline Variables & Coeficiente & $\begin{array}{c}\text { Error } \\
\text { estándar }\end{array}$ & Valor t & Valor $\boldsymbol{P}$ \\
\hline $\mathrm{X}_{1}$ Zona & 0.031 & 0.012 & 2.587 & 0.010 \\
X $_{2}$ Tamaño A & -0.002 & 0.003 & -0.670 & 0.503 \\
X $_{3}$ Tamaño B & 0.001 & 0.003 & 0.228 & 0.820 \\
X $_{4}$ Sistema de identificación & 0.023 & 0.002 & 9.895 & 0.000 \\
X $_{5}$ Género & -0.001 & 0.003 & -0.328 & 0.743 \\
X $_{6}$ Ubicación e instalación del apiario & -0.094 & 0.007 & -13.033 & 0.000 \\
X $_{7}$ Alimentación artificial & -0.059 & 0.010 & -6.005 & 0.000 \\
X $_{8}$ Sanidad apícola & -0.086 & 0.009 & -9.762 & 0.000 \\
X $_{9}$ Materiales protección colmena & -0.119 & 0.006 & -20.604 & 0.000 \\
X $_{10}$ Cosecha & -0.114 & 0.004 & -26.611 & 0.000 \\
X $_{11}$ Personal en campo & -0.178 & 0.005 & -35.644 & 0.000 \\
X $_{12}$ Programa de limpieza e higiene & -0.069 & 0.004 & -18.700 & 0.000 \\
\hline $\mathrm{R}^{2}=0.393$ & & & &
\end{tabular}


$\dot{\mathrm{R}}^{2}$ corregida $=0.392$

F estadística $=435.578$

Sig. Cambio en $\mathrm{F}=0.000$

Variable dependiente: Dif_InABPPM.

De igual manera, las pruebas de $t$ de los parámetros del modelo señalan que solo las variables tamaño $\left(\mathrm{X}_{2}\right.$ y $\left.\mathrm{X}_{3}\right)$ y género $\left(\mathrm{X}_{5}\right)$ no son significativas $(P>0.1)$. Con relación a la zona $\left(\mathrm{X}_{1}\right)$, destaca que el InABPPM tiende a presentar mayores incrementos en las zonas urbanas con respecto a las áreas rurales $(P<0.05)$. Asimismo, el contar con un sistema de identificación $\left(\mathrm{X}_{4}\right)$ de apiarios influye $(P<0.01)$ en el incremento del InABPPM; esto se puede explicar porque la intervención de un programa de BPPM riguroso requiere llevar registros en bitácoras por parte de los apicultores.

Finalmente, las variables relacionadas con las categorías del catálogo de innovaciones ( $\mathrm{X}_{6}$, $\mathrm{X}_{7}, \mathrm{X}_{8}, \mathrm{X}_{9}, \mathrm{X}_{10}, \mathrm{X}_{11}$ y $\left.\mathrm{X}_{12}\right)$, resultaron significativas en el modelo $(P<0.01)$; destacando el hecho de que el coeficiente de regresión tiene un efecto negativo, e indica que a mayor adopción en ELB, el incremento en la adopción es menor y viceversa. Resultado que concuerda con el análisis univariado presentado anteriormente.

\section{Niscusión V}

La producción de alimentos en el mundo enfrenta exigencias cada vez más estrictas en los mercados nacionales y de exportación ${ }^{(24)}$, para garantizar el cumplimiento de la normatividad de proceso a lo largo de la cadena de valor, los actores privados han desarrollado esquemas de certificación e integración vertical ${ }^{(25)}$. La producción de miel no está exenta de estas restricciones; en respuesta a los requerimientos que exige el mercado, las autoridades del sector apícola en México han optado por promover la adopción de BPPM encaminadas a lograr la producción de miel con altos estándares de calidad e inocuidad ${ }^{(5)}$.

El InABPPM por tipo de apicultor y categoría, indica que los pequeños apicultores son los que más incrementan sus niveles de adopción en comparación con los medianos y grandes; situación similar a lo reportado con apicultores del estado de Morelos, México, donde se encontró que apicultores pequeños con nivel tecnológico bajo usan menos tecnología en el manejo, alimentación y genética que los apicultores medianos y grandes con nivel tecnológico intermedio ${ }^{(26)}$. Por el contrario, otros autores han reportado que en cultivos de 
frambuesa los pequeños agricultores son menos propensos a poner en práctica las normas de seguridad y calidad alimentaria; sin embargo, una vez que son capaces de superar las barreras, ellos mismos son espectadores del efecto positivo en el rendimiento, calidad y utilidad del producto $^{(24)}$. Por su parte, Reardon et $a l^{(27)}$ indican que aunque la actualización y cumplimiento de estándares implica beneficios sustanciales en términos de mejora en los precios y acceso a los mercados, se teme que los productores de pequeña escala podrían ser excluidos por los costos adicionales implicados. En específico, los resultados encontrados con los apicultores de la Península de Yucatán en México, se explican por el acceso al programa de capacitación a través de la difusión e implementación de BPPM y la implementación del modelo de gestión de la innovación vía las AGI-DP.

Sin embargo, el costo de las certificaciones en cumplimiento de calidad es elevado para los productores agropecuarios de pequeña escala, por lo que a menudo tienen que depender de la ayuda de agentes externos de la cadena de suministro (instituciones gubernamentales y no gubernamentales), a través de programas de extensionismo especializados, de lo contrario varios de ellos podrían ser excluidos de los mercados de exportación debido a la creciente importancia de las normas de calidad. En el caso analizado, el trabajo de los extensionistas, agrupados en las AGI-DP, fue pagado con recursos públicos, en apoyo a los apicultores de la Península de Yucatán.

Aunado a lo anterior, la implementación de BPPM, las normas y certificación de calidad trae consigo posibles cambios directos como el acceso a las cadenas de valor modernas en los mercados nacionales e internacionales, y los precios de venta más altos ${ }^{(28)}$, así como un uso más eficiente de los insumos, mejores condiciones de higiene y salud, mejora de la calidad del suelo, de la calidad del producto y el aumento o estabilidad del rendimiento, que con el tiempo también puede dar lugar a mayores ingresos ${ }^{(29,30)}$.

En este estudio se identificó que después de la intervención del programa de extensión, las categorías con mayor adopción fueron las de ubicación e instalación del apiario, sanidad apícola, y personal de campo, en respuesta a los requerimientos de calidad exigidos por el mercado nacional y extranjero. Al respecto, Wei et al $l^{(31)}$ indican que los países importadores de miel han implementado medidas sanitarias y fitosanitarias a través de continuas notificaciones a la Organización Mundial del Comercio, en consecuencia la posición de los países exportadores de miel en el mundo es variable.

De la misma forma, Formato y Smulders ${ }^{(32)}$ mencionan que prácticas como ubicar las colmenas en áreas agrícolas tienen efectos importantes en la salud de la colmena, la calidad de la miel y la eficiencia productiva. También señalan que las buenas prácticas relacionadas con el manejo de medicamentos autorizados para el control sanitario, la frecuencia de revisión de las colmenas, y las prácticas sobre alimentación artificial también tienen efectos muy importantes en la calidad de la miel, la salud de la colmena, la seguridad biológica de la miel como alimento y la eficiencia productiva. 
Con relación a la sanidad apícola, las principales innovaciones difundidas fueron: i) la BP13, frecuencia revisión de las colmenas; ii) BP15, uso de productos autorizados para prevenir enfermedades; y iii) BP17, control de la varroasis. Con referencia a esta última, Giacobino et $a l^{(33)}$ resaltan el daño ocasionado por la varroa, pues se considera una de las principales amenazas para la apicultura en todo el mundo; el daño causado por el ácaro influye en la pérdida de peso corporal, malformaciones y el debilitamiento de las abejas. Además, estos autores destacan que el uso de los estudios epidemiológicos proporciona información clave para el diseño de programas de vigilancia. También, la ubicación de los apiarios en diferentes regiones y las condiciones climáticas influyen en el nivel de infestación de varroa. Lo anterior permite resaltar la importancia de la continuidad de los esquemas de atención a productores apícolas en México, dado que las áreas de oportunidad requieren ser atendidas desde diversas vertientes.

Del modelo de regresión se destaca principalmente que la variable género no influye en el incremento del InABPPM. Con relación al estrato del productor por número de colmenas, en los resultados no se encontró significancia $(P>0.10)$, resultados similares reportaron otros autores, quienes encontraron que el tamaño del apiario, medido en el número de colmenas, no tuvo efectos significativos en la adopción de tecnologías apícolas en Uganda ${ }^{(34)}$. Aunque aparentemente no hay efecto del tamaño del apiario en la adopción de BPPM, sí se han reportado efectos de esta variable en el ingreso de los apicultores en países como Etiopía ${ }^{(30)}$. Sin embargo, cabe destacar nuevamente, que el análisis univariado sí demostró que la adopción es mayor en apicultores pequeños.

En sentido contrario, la ubicación del apiario influye en el incremento de la adopción de BPPM, siendo la zona urbana la que tiende a incrementar en mayor grado, lo cual se puede explicar por su cercanía a organismos gubernamentales y privados, así como con otro tipo de actores, por ejemplo, proveedores de insumos, quienes figuran como complementadores al momento de obtener información para la adopción de las prácticas, así como un mayor acceso a los insumos.

Por otro lado, la alimentación artificial resultó ser una variable significativa en el incremento del nivel de adopción de las innovaciones, destacando la BP10 sobre el uso de insumos permitidos por la SAGARPA como fuentes de energía al preparar el alimento artificial (azúcar, barredura, jarabe de fructosa, piloncillo, miel), y BP11 los insumos permitidos por la SAGARPA como complementos; esto puede tener otros efectos positivos, pues como lo señalan Giacobino et $a l^{(33)}$, cuando la dieta de proteína se basa en el suplemento de polen natural, ésta influye en la disminución hasta de $3 \%$ de infestación de varroa, ya que se consume con mayor facilidad que otros sustitutos, además, aumenta el tamaño de la colonia y diluye la población de ácaros. Por otro lado, el uso de jarabe de maíz con fructosa como fuente de hidratos de carbono se asocia con una menor prevalencia de infestación del ácaro. 
Las categorías sanidad apícola, materiales para la protección de la colmena, el personal de campo y el programa de limpieza e higiene influyeron también en el incremento del InABPPM, y en la obtención de miel de calidad, ya que las medidas de higiene, en particular el tratamiento de las colonias de abejas y desinfección de los equipos por parte del personal de campo, son tareas obligatorias para garantizar la rápida erradicación de cualquier brote de una enfermedad ${ }^{(33)}$.

Cabe señalar también que la ubicación de los apiarios por zona geográfica, el clima y las condiciones ambientales influyen en la tasa de crecimiento de los ácaros e implica mayor tiempo de flujo climático y periodos más largos de cría, reflejados en la calidad de la miel $^{(35,36)}$.

Además del modelo de extensión utilizado, existen otros factores que pueden estar influyendo en la adopción de BPPM y que valdría la pena profundizar más en el análisis de estas causas en futuras investigaciones. Por ejemplo, en Kenya, utilizaron un modelo de regresión, donde señalan que factores como la escolaridad, el ingreso mensual y la frecuencia de visitas al apiario influyen de manera positiva en la adopción de tecnologías apícolas modernas ${ }^{(37)}$. Adgaba et $a l^{(38)}$ también reportan efecto positivo de la escolaridad y el tamaño de la familia en la adopción de tecnologías apícolas en Arabia Saudita, así como una relación negativa con la edad del apicultor. También se han reportado otras variables que tienen efecto en la adopción de BPPM, como el acceso a créditos y servicios de extensionismo ${ }^{(34)}$.

En esta investigación se valoró el incremento del InABPPM en tres estados productores de acuerdo a la zona (rural o urbana), dejando pendiente el análisis del nivel de adopción que considere las características propias de la región. Se encontró que cada uno de los estados evaluados adoptó en mayor o menor medida ciertas categorías. En el caso de Yucatán la sanidad apícola, cosecha, personal en campo y programa de higiene y limpieza; en Quintana Roo la ubicación e instalación del apiario y la alimentación artificial; y finalmente los apicultores de Campeche adoptaron las BPPM de la categoría de materiales para la protección y ahumado de la colmena. Este tipo de resultados pueden tener implicaciones de interés, porque es importante difundir aquellas prácticas e innovaciones que sean adecuadas a las condiciones de cada estado, lo cual ha sido indicado por otros autores ${ }^{(12)}$.

\section{《 Conclusiones e implicaciones $\|$}


El análisis del cambio en la adopción de BPPM por efecto de un programa de asistencia técnica y capacitación, refleja que los aumentos en las BPPM de proceso y de organización presentan valores mayores en comparación con aquéllas clasificadas como de producto; estas últimas requieren mayor inversión para implementarlas, lo cual limita su adopción. Este estudio proporciona mayor evidencia con relación a las estrategias de fomento de diversas innovaciones más fáciles y baratas de adoptar. En este escenario, convendría impulsar las BPPM de proceso, y posteriormente orientar los apoyos gubernamentales a la inversión en los apiarios con mejor nivel de adopción de ellas, con lo cual también se contribuiría a hacer más eficiente el gasto público. Los servicios de extensión presentan un efecto positivo en la adopción de BPPM, pero es necesario considerar las diferencias en los territorios evaluados. Dichas diferencias pueden obedecer a aspectos ambientales propiamente, pero sin duda también pueden deberse al arreglo socioeconómico e institucional que prevalece en los estados estudiados. Existen al menos dos procesos críticos para lograr que una producción de miel cumpla las normas de calidad que exige el mercado internacional: primero, se debe producir con niveles altos de adopción de BPPM; segundo, los centros de acopio, los intermediarios y la propia agroindustria debe también observar altos niveles de adopción de buenas prácticas de manufactura. Esta investigación aporta elementos para ser considerados en el proceso primario de la producción de miel, pero es necesario investigar con mayor detalle lo que ocurre con la miel al ingresar al eslabón agroindustrial de la cadena productiva. Lo anterior implica mejoras considerables en la implementación de protocolos de trazabilidad, para ofrecer garantías a los compradores nacionales y extranjeros.

\section{- Agradecimientos}

A los apicultores, al Instituto Nacional para el Desarrollo de Capacidades del Sector Rural (INCA-Rural), la UTE-Innovación y al CIESTAAM por proporcionar la información para realizar la presente investigación. Parte de la investigación se desarrolló con apoyo económico del proyecto 650 de la Dirección General de Investigación y Posgrado de la Universidad Autónoma Chapingo.

\section{- Literatura citada}

1. SAGARPA. Miel. Atlas agroalimentario. Secretaría de Agricultura, Ganadería, Desarrollo Rural, Pesca y Alimentación (SAGARPA). 2016. 
2. Magaña MMA, Sanginés GJR, Lara y Lara P, Salazar BL de L, Leyva MCE. Competitividad y participación de la miel mexicana en el mercado mundial. Rev Mex Cienc Pecu 2017;8(1):43-52.

3. Martínez GEG, Pérez LH. La producción de miel en el trópico húmedo de México: avances y retos en la gestión de la innovación. Texcoco, Estado de México: Universidad Autónoma Chapingo-CIESTAAM. 2013.

4. Villanueva-Gutiérrez R, Echazarreta-González C, Roubik DW, Moguel-Ordóñez YB. Transgenic soybean pollen (Glycine max L.) in honey from the Yucatán peninsula, Mexico. Sci Rep 2014;4:4022.

5. SAGARPA, SENASICA, Coordinación General de Ganadería, Sistema Producto Apícola. Manual de buenas prácticas pecuarias en la producción de miel. Tercera ed. México, DF. Secretaría de Agricultura, Ganadería, Desarrollo Rural, Pesca y Alimentación (SAGARPA). Servicio Naciona de Sanidad, Inocuidad y Calidad Agroalimentaria (SENASICA). 2015.

6. Alfaro BRG, Gonzales AJA, Ortiz DJJ, Viera CFA, Burgos PAI, Martínez HE, et al. Caracterización palinológica de las mieles de la Península de Yucatán. Mérida, Yucatán: Universidad Autónoma de Yucatán; Comisión Nacional para el Conocimiento y Uso de la Biodiversidad. 2010.

7. Aguilar-Ávila J, Santoyo-Cortés VH, Muñoz-Rodríguez M, Aguilar-Gallegos N, Martínez-González EG. Agencias de gestión de la innovación para el desarrollo de proveedores en México. Compés LR, et al, editores. Redes de innovación y desarrollo local para el medio rural. Gobierno de España; Ministerio de Agricultura, Alimentación y Medio Ambiente; 2015:249-266.

8. Sagastume N, Obando M, Martínez M. Guía para la elaboración de estudios de adopción de tecnologías de manejo sostenible de suelos y agua. Primera ed. Tegucigalpa, Managua y San Salvador: PASOLAC, inter-cooperation, COSUDE; 2006.

9. Rogers EM. Diffusion of innovations. Fifth ed. New York, USA: Free Press; 2003.

10. Hartwich F, Monge PM, Ampuero RL, Soto JL. Knowledge management for agricultural innovation: lessons from networking efforts in the Bolivian Agricultural Technology System. Knowl Manag Dev J 2007;3(2):21-37.

11. Aguilar-Gallegos N, Martínez-González EG, Aguilar-Ávila J, Santoyo-Cortés H, Muñoz-Rodríguez M, García-Sánchez EI. Análisis de redes sociales para catalizar la innovación agrícola: de los vínculos directos a la integración y radialidad. Est Gerenciales 2016;32(140):197-207.

12. Aguilar-Gallegos N, Muñoz-Rodríguez M, Santoyo-Cortés H, Aguilar-Ávila J, Klerkx L. Information networks that generate economic value: A study on clusters of adopters of new or improved technologies and practices among oil palm growers in Mexico. Agricult Syst 2015;135:122-32. 
13. Kilelu CW, Klerkx L, Leeuwis C. Unravelling the role of innovation platforms in supporting co-evolution of innovation: Contributions and tensions in a smallholder dairy development programme. Agricult Syst 2013;118:65-77.

14. Klerkx L, Aarts N, Leeuwis C. Adaptive management in agricultural innovation systems: The interactions between innovation networks and their environment. Agricult Syst 2010;103(6):390-400.

15. Jara-Rojas R, Bravo-Ureta BE, Díaz J. Adoption of water conservation practices: A socioeconomic analysis of small-scale farmers in Central Chile. Agricult Syst 2012;110:54-62.

16. Mariano MJ, Villano R, Fleming E. Factors influencing farmers' adoption of modern rice technologies and good management practices in the Philippines. Agricult Syst 2012;110:41-53.

17. Güemes-Ricalde FJ, Villanueva-G R, Eaton KD. Honey production by the Mayans in the Yucatan peninsula. Bee World 2003;84(4):144-54.

18. Contreras-Escareño F, Armendáriz BP, Echazarreta CM, Arroyo JC, Macías-Macías JO, Tapia-González JM. Características y situación actual de la apicultura en las regiones Sur y Sureste de Jalisco, Mexico. Rev Mex Cienc Pecu 2013;4(3):387-398.

19. Muñoz RM, Aguilar ÁJ, Rendón MR, Altamirano CJR. Análisis de la dinámica de innovación en cadenas agroalimentarias. Texcoco, Estado de México: Universidad Autónoma Chapingo-CIESTAAM; 2007.

20. OCDE Organisation for Economic Co-operation and Development. Creating rural indicators for shaping territorial policy. OECD Organisation for Economic Co-operation and Development. 1994.

21. INEGI Instituto Nacional de Estadística y Geografía. Censo de población y vivienda. 2010.

22. Montgomery DC, Peck EA, Vinning GG. Introducción al análisis de regresión lineal. Segunda ed. México, D F: Compañia editorial continental; 2002.

23. SAS. SAS/STAT (R). User's guide. Cary, N.C., USA: Statistical Analysis System; 2004.

24. Handschuch C, Wollni M, Villalobos P. Adoption of food safety and quality standards among Chilean raspberry producers - Do smallholders benefit?. Food Policy 2013;40:64-73.

25. Reardon T, Codron J-M, Busch L, Bingen J, Harris C. Global change in agrifood grades and standards: agribusiness strategic responses in developing countries. Int Food Agribus Manag Rev 1999;2(3-4):421-35.

26. Vélez IA, Espinosa GJA, Amaro GR, Arechavaleta VME. Tipología y caracterización 
de apicultores del estado de Morelos, México. Rev Mex Cienc Pecu 2016;7(4):507-24.

27. Reardon T, Barrett CB, Berdegué JA, Swinnen JFM. Agrifood industry transformation and small farmers in developing countries. World Dev 2009;37(11):1717-27.

28. Asfaw S, Mithöfer D, Waibel H. EU food safety standards, pesticide use and farm-level productivity: the case of high-value crops in Kenya. J Agric Econ 2009;60(3):645-667.

29. Hobbs J. Incentives for the adoption of good agricultural practices. Rome, Italy: Food and Agriculture Organization of the United Nations (FAO); 2007.

30. Girma J, Gardebroek C. The impact of contracts on organic honey producers' incomes in southwestern Ethiopia. For Policy Econ 2015;50:259-68.

31. Wei G, Huang J, Yang J. Honey safety standards and its impacts on China's honey export. J Integr Agric 2012;11(4):684-693.

32. Formato G, Smulders FJM. Risk management in primary apicultural production. Part 1: bee health and disease prevention and associated best practices. Vet Q 2011;31(1):2947.

33. Giacobino A, Cagnolo NB, Merke J, Orellano E, Bertozzi E, Masciangelo G, et al. Risk factors associated with the presence of Varroa destructor in honey bee colonies from east-central Argentina. Prev Vet Med 2014;115(3-.4):280-287.

34. Mujuni A, Natukunda K, Kugonza DR. Factors affecting the adoption of beekeeping and associated technologies in Bushenyi District, Western Uganda. Livest Res Rural Dev 2012;24(8). http://www.lrd.org/lrrd24/8muju24133.htm. Accessed Apr 10, 2016.

35. Mattila HR, Otis GW. Influence of pollen diet in spring on development of honey bee (Hymenoptera: apidae) colonies. J Econ Entomol 2006;99(3):604-613.

36. Fechner DC, Moresi AL, Ruiz DJD, Pellerano RG, Vazquez FA. Multivariate classification of honeys from Corrientes (Argentina) according to geographical origin based on physicochemical properties. Food Biosci 2016;15:49-54.

37. Affognon HD, Kingori WS, Omondi AI, Diiro MG, Muriithi BW, Makau S, et al. Adoption of modern beekeeping and its impact on honey production in the former Mwingi District of Kenya: assessment using theory-based impact evaluation approach. Int J Trop Insect Sci 2015;35(2):96-102.

38. Adgaba N, Al-Ghamdi A, Shenkute AG, Ismaiel S, Al-Kahtani S, Tadess Y, et al. Socioeconomic analysis of beekeeping and determinants of box hive technolgy adoption in the Kingdom of Saudi Arabia. J Anim Plant Sci 2014;24(6):1876-1884. 